\title{
Diagnosis and treatment of pulmonary mucormycosis: A case report
}

\author{
ZHIMING LUO and LIN ZHANG \\ Department of Respiratory Medicine, Xuan Wu Hospital, Capital Medical University, Beijing 100053, P.R. China
}

Received June 16, 2016; Accepted May 5, 2017

DOI: $10.3892 /$ etm.2017.4986

\begin{abstract}
Pulmonary mucormycosis, a relatively rare pulmonary fungal disease, is difficult to diagnose and lacks effective treatment. The present study reports the case of a 64-year-old patient who was treated successfully for pulmonary mucormycosis in Xuan Wu Hospital. The patient presented with low-grade fever and a productive cough that persisted for 1 month with no evident cause, and also suffered from diabetes mellitus. Prior antibiotic treatment with levofloxacin had been ineffective. Culture of mucus obtained by bronchoscopy resulted in a diagnosis of pulmonary mucormycosis. The patient received a cumulative dose of $1,355 \mathrm{mg}$ amphotericin $\mathrm{B}$ over 2 months and presented a full recovery.
\end{abstract}

\section{Introduction}

With the increasing use of broad-spectrum antibiotics, chemicals, immunosuppressive agents and organ transplantation, the incidence of invasive pulmonary fungal infections has increased in recent years. A retrospective investigation of 16 hospitals organized by the Chinese Medical Association Respiratory Branch demonstrated that, among 474 cases of invasive pulmonary fungal infections, the four leading diagnoses were pulmonary aspergillosis, pulmonary candidiasis, pulmonary coccidioidomycosis and pulmonary mucormycosis (1). Pulmonary mucormycosis, a comparatively rare form of pneumomycosis, is difficult to diagnose and lacks effective treatment methods. The incidence of pulmonary mucormycosis ranks only second to that of nose and cerebral mucormycosis (2). Susceptible individuals inhale fungal spores in the air or paranasal sinus, resulting in pulmonary mucormycosis via blood-borne or lymphatic dissemination. The clinical characteristics of pulmonary mucormycosis are non-specificity pneumonia. The most common symptom persistent high fever $\left(>38^{\circ} \mathrm{C}\right)$, cough, hemoptysis and chest pain (3).

Correspondence to: Dr Zhiming Luo, Department of Respiratory Medicine, Xuan Wu Hospital, Capital Medical University, 45 Changchun Street, Xicheng, Beijing 100053, P.R. China E-mail: 13718962892@163.com

Key words: pulmonary mucormycosis, diagnosis, treatment
Tedder's analysis of 255 pulmonary mucormycosis revealed that $37 \%$ of patients suffer from hematological malignancies, $32 \%$ of patients suffer from diabetes and $31 \%$ of patients suffer from chronic renal failure and organ transplantation (4). The patients with solid tumor seldom suffer from secondary pulmonary mucormycosis (5). Amphotericin B is polyene antifungal drug, and is the selected drug for the treatment of mucormycosis (6). Different mucormycosis exhibit different susceptibility to Amphotericin B, and the drug concentration of Amphotericin B in the lung is lower than other tissues. Therefore, pulmonary mucormycosis infection requires a higher dose of Amphotericin B. The accumulated dose can reach $30 \mathrm{mg} / \mathrm{kg}$ and the total dose can reach 2,500-3,000 mg for the total treatment course of 1-3 months. Previously, a study has suggested that Liposome amphotericin B was safer, with an improved effect and smaller accumulated dose, compared with amphotericin B alone (7). Singh's study indicated that, in addition to the Amphotericin B with good effect, antifungal drugs, such as itraconazole and voriconazole, may be a clinical option (8). For the patients who do not tolerate the traditional drug or with whom the drug exhibited no effect, novel triazole fungicide posaconazole is better than voriconazole and fluconazole (9). Certain reports indicate that Echinocandins may be used as a drug combination $(10,11)$. Due to mucormycosis causing angiemphraxis, the drug may not reach the focus of infection, thus, when the antifungal drug has no good effect, pulmonary lobectomy was suggested (12). This disease progresses slowly and the fatality rate has been reported to be high. The present study reports the details of the diagnosis and therapy of a case with pulmonary mucormycosis that was successfully treated with amphotericin B.

\section{Case report}

A 64-year-old man with a 1-month history of low-grade fever and productive cough of no apparent cause presented to the Xuan Wu Hospital (Beijing, China) in April 2014. The patient suffered from diabetes mellitus and chronic bronchitis for 3 years, and had no history of allergies. The temperature of the patient was $37.5^{\circ} \mathrm{C}$ upon admission, and he was expectorating $\sim 20 \mathrm{ml}$ of yellow or white sputum daily. Fasting blood glucose concentration was 7-9 mmol/1 (normal reference ranges 6-7.1 mmol/l). Initially, a pulmonary infection was diagnosed at the outpatient department according to a chest computed tomography (CT) scan, and the patient received antibiotic 
treatment with intravenous levofloxacin, $400 \mathrm{mg}$ once a day, for 7 days. However, the symptoms did not improve, while breathing became more difficult, and the patient reported difficulties in climbing two flights of stairs. Therefore, the patient was admitted to Xuan Wu Hospital.

On admission, a pulse rate of 89 beats/min, normal heart sounds, a respiratory rate of 22 breaths/min and blood pressure of 100/60 mmHg were reported. The patient was alert, oriented and in good general condition. Pulmonary breath sounds were faint bilaterally, and there were intermittent fine moist rales over the two lower lobes. The abdomen was soft, with no tenderness or rebound tenderness. The lower limbs were not edematous

Laboratory investigations while breathing normally revealed the following: $\mathrm{pH}$ of arterial blood, 7.42 (normal reference ranges 7.35-7.45); partial $\mathrm{O}_{2}$ pressure $\left(\mathrm{PO}_{2}\right), 72 \mathrm{mmHg}$ (normal reference ranges $80-100 \mathrm{mmHg}$ ); partial $\mathrm{CO}_{2}$ pressure $\left(\mathrm{PCO}_{2}\right), 45 \mathrm{mmHg}$ (normal reference ranges 35-45 mmHg); white blood cell (WBC) count, 8.46x10 $/ 1$ (normal reference ranges $4.0-10.0 \times 10^{9} / 1$ ); neutrophils $64.3 \%$ (normal reference ranges 45-70\%); and hemoglobin $(\mathrm{Hb}), 111 \mathrm{~g} / 1$ (normal reference ranges $110-160 \mathrm{~g} / \mathrm{l})$. In addition, routine urine tests were positive for albumin (ALB) and urine sugar. Biochemical tests indicated the following results: Serum ALB level, $30.9 \mathrm{~g} / \mathrm{l}$ (normal reference ranges $>35 \mathrm{~g} / \mathrm{l}$ ); prealbumin (PAB) level, $101 \mathrm{mg} / \mathrm{l}$; blood glucose, $8.26 \mathrm{mmol} / 1$ (normal reference ranges 6-7.1 mmol/l); ESR, $58 \mathrm{~mm} / 1$ (normal reference ranges $<20 \mathrm{~mm} / \mathrm{l}$ ); and procalcitonin, $0.081 \mathrm{ng} / \mathrm{ml}$ (normal reference ranges $<0.5 \mathrm{ng} / \mathrm{ml}$ ). Microscopic examination of a sputum smear demonstrated moderate numbers of gram-positive cocci and streptococci, as well as occasional gram-negative bacilli; however, no fungal spores or pseudohyphae were observed. Sputum culture and acid-fast staining were negative, while the cancer antigen 125 level was $44.04 \mu \mathrm{g} / \mathrm{ml}$. A chest CT scan demonstrated poorly defined flocculant shadowing in the right lower and in the left lower lobe, and macular lesions in right lower lobe were observed (Fig. 1). Lung function tests showed that the forced expiratory volume in $1 \mathrm{sec}(\mathrm{FEV} 1) /$ forced vital capacity was $53 \%$, with the FEV1 being $61 \%$ of the predicted value total lung capacity.

According to the observations, the patient was initially diagnosed with community-acquired pneumonia and chronic obstructive pulmonary disease, and was treated with $2,000 \mathrm{mg}$ moxalactam (Hailing Pharmaceutical Company, Ltd., Hainan, China), twice a day and $400 \mathrm{mg}$ moxifloxacin (Bayer Schering Pharma AG, Berlin, Germany) once a day via intravenous drip for two weeks. The patient's temperature fluctuated at $\sim 37.5^{\circ} \mathrm{C}$ for 2 weeks, and he continued to produce small amounts of yellow, occasionally blood-stained sputum and to complain of shortness of breath with activity. Two weeks after the initiation of treatment, repeat laboratory tests provided the following results: WBC count, 9.05×10\%/l; neutrophils, 67.8\%; Hb level, $111 \mathrm{~g} / \mathrm{l}$; ALB, $30.46 \mathrm{~g} / \mathrm{l}$; PAB, $106 \mathrm{mg} / \mathrm{l}$; creatinine, $62 \mathrm{~mol} / \mathrm{l}$; potassium level, $4.04 \mu \mathrm{mol} / 1$; procalcitonin, $0.05 \mathrm{ng} / \mathrm{ml}$. In addition, blood gas analysis in room air produced the following findings: $\mathrm{pH}, 7.427 ; \mathrm{PO}_{2}, 53.6 \mathrm{mmHg}$; and $\mathrm{PCO}_{2}, 38.6 \mathrm{mmHg}$. Galactomannan and $\beta$-D-glucan detection, latex agglutination tests, T-SPOT test for tuberculosis, and anti-nuclear and anti-neutrophil cytoplasmic antibody detection all provided negative results. A further chest CT scan, performed three

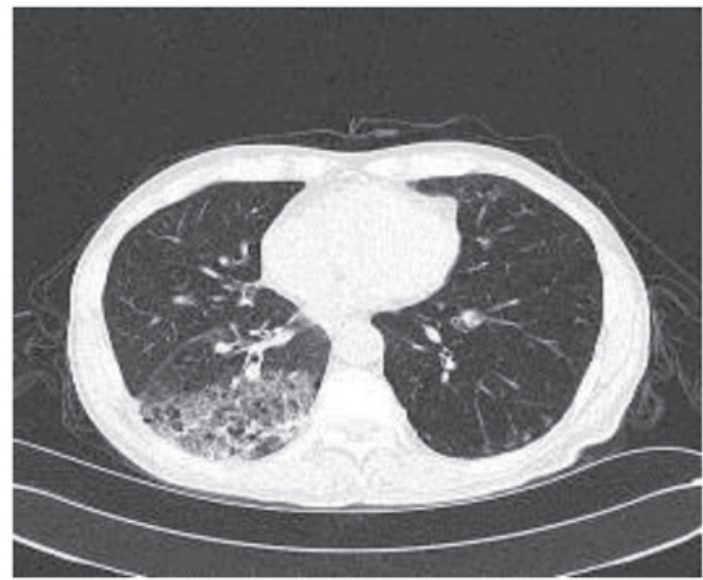

Figure 1. Plain computed tomography scan upon admission to the hospital, demonstrating patchy and macular lesions in the patient's bilateral pulmonary lobe, and macular lesions in right lower lobe were observed.

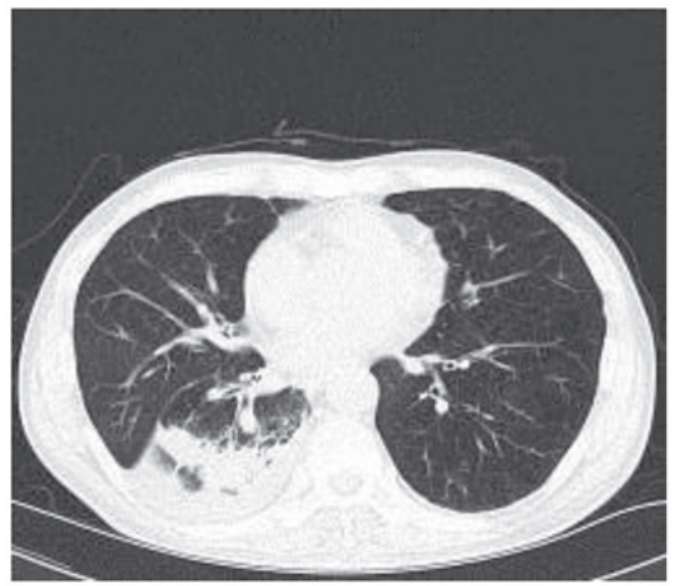

Figure 2. Plain computed tomography scan performed three weeks after the initiation of treatment. Consolidation was observed in the right lower lobe of the patient. This suggests that the antibiotic treatment was ineffective and further etiological examination was required.

weeks after initiation treatment demonstrated that the right middle lobe lesions had resolved, however, the right lower lobe lesions were still present (Fig. 2).

Three weeks after the initiation treatment, bronchoscopy displayed bilateral bronchial mucous membrane hyperemia and a small amount of sticky secretions in the right lower lobe lumens. A photomicrograph of crystal violet-stained fungal colony cultured from mucus collected by bronchoscopy indicated that the hyphae and spores typical of the Mucor genus are present, following Medan phenol staining and imaging with an optical microscope at a magnification of $x 400$ (Fig. 3). Therefore, the patient was diagnosed with mucormycosis. He was treated with $5 \mathrm{mg}$ daily amphotericin B (Huabei Pharmaceuticals Ltd., Hebei, China), with the dose later increased from $5 \mathrm{mg}$ per day to $0.5 \mathrm{mg} / \mathrm{kg} /$ day to $30 \mathrm{mg}$ per day, over 2 weeks. A plain computed tomography image was obtained 2 weeks after commencing amphotericin B treatment, demonstrating partial resolution of consolidation in the patient's right lower lobe (Fig. 4). A total dose of $1,355 \mathrm{mg}$ was administered over 2 months, plain computed 


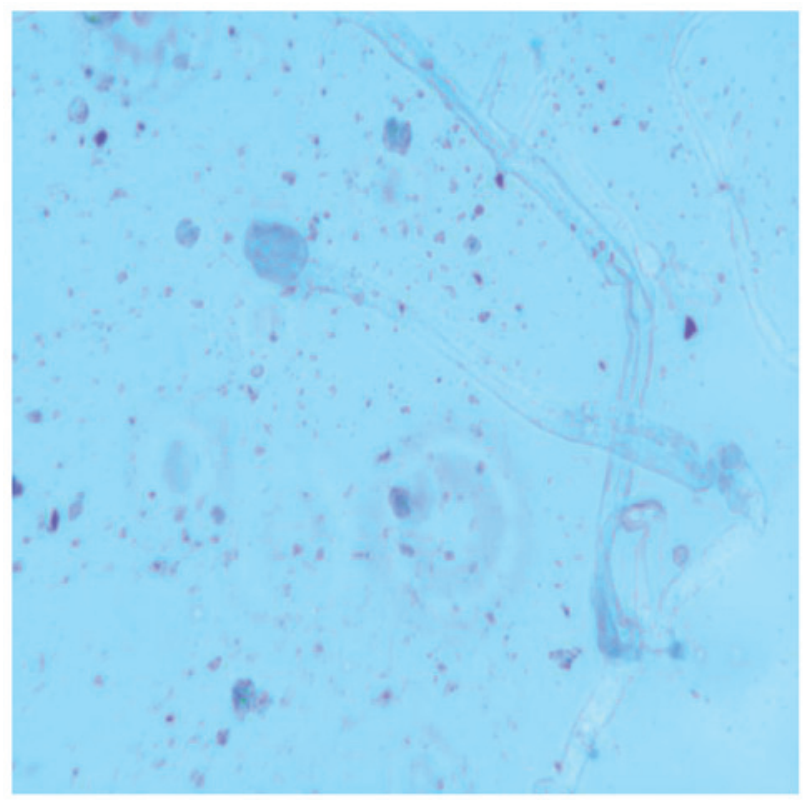

Figure 3. Photomicrograph of crystal violet-stained fungal colony cultured from mucus collected by bronchoscopy. The analysis demonstrated hyphae and spores typical of the Mucor genus under a Medan phenol staining optical microscope (magnification, $\mathrm{x} 400$ ).

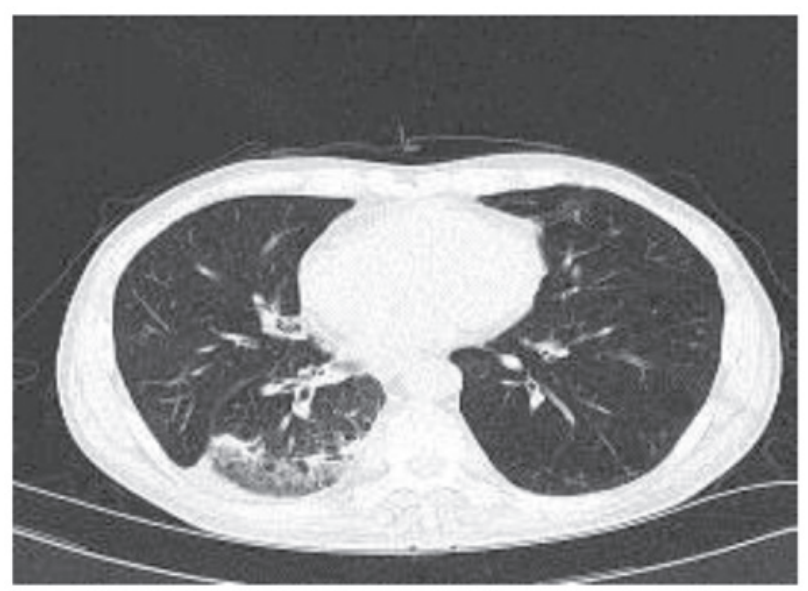

Figure 4. Plain computed tomography image obtained 2 weeks after commencing amphotericin B treatment, demonstrating partial resolution of consolidation in the patient's right lower lobe.

tomography image on completion of two months treatment, revealing almost complete resolution of consolidation in the patient's right lower lobe (Fig. 5). The patient made a complete recovery and was discharged from the hospital. After hospital discharge, the patient had no any corresponding treatment administered. One month later, the patient returned to hospital and completed the assessments. The patient had no complaints, and the blood routine examination, renal function and serum potassium were within the normal range. WBC count, $8.05 \times 10^{9} / 1$; neutrophils, $63.8 \%$; Hb level, $121 \mathrm{~g} / 1$, creatinine $49 \mu \mathrm{mol} / 1$ (normal reference ranges 53-106 $\mu \mathrm{mol} / \mathrm{l}$ ), urea nitrogen $6.4 \mathrm{mmol} / \mathrm{l}$ (normal reference ranges 3.2-7.1 $\mathrm{mmol} / \mathrm{l}$ ), potassium level, $3.9 \mu \mathrm{mol} / \mathrm{l}$; Written informed consent was obtained from the patient prior to participation in the present study.

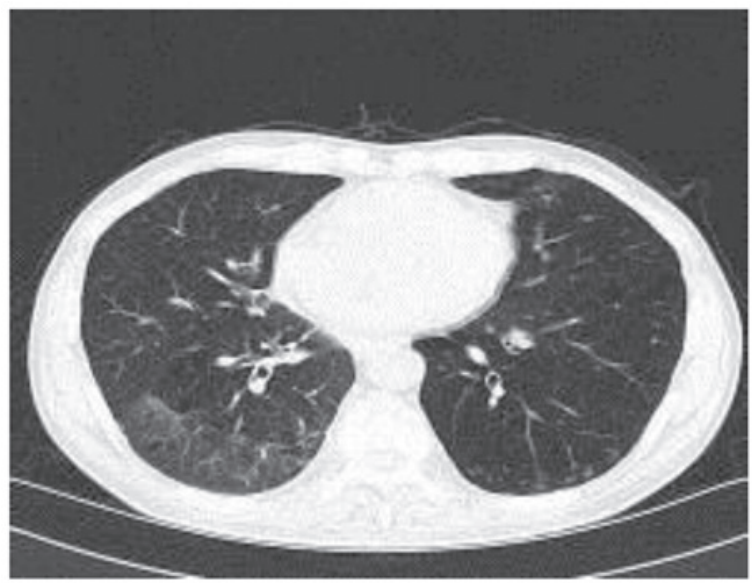

Figure 5. Plain computed tomography image on completion of two months treatment, revealing almost complete resolution of consolidation in the patient's right lower lobe.

\section{Discussion}

Fungi of the Mucor genus are conditional pathogens that rarely cause disease in healthy individuals. However, inhalation of these spores may result in disease in subjects with impaired immune function (13). The organisms spread through the paranasal sinuses and respiratory system, or by the hematogenous or lymphatic route (3). The majority of patients with mucormycosis present one or more risk factors, including hematopoietic malignancies, treatment with immune inhibitors or diabetes mellitus. In addition, a low neutrophil count is an independent risk factor for Mucor infection (14). Pulmonary mucormycosis accounts for $25 \%$ of cases of mucormycosis, followed by nasal and brain mucormycosis. Furthermore, pulmonary mucormycosis has a fatality rate of $>50 \%$ (2). Pulmonary mucormycosis develops quickly and is characteristically a subacute condition. The clinical manifestations are non-specific and commonly include fever, cough, chest pain, dyspnea and hemoptysis, since these pathogens can erode blood vessels (15). Radiological manifestations include infiltrates, exudation, consolidation, cavities and nodules, while the disease typically has a predilection for the upper lobes $(3,16)$. The present study presented with an unusual characteristic of the disease manifesting in the lower lobes, which has previously been unobserved. The reason for this manifestation remains unclear and future studies should assess it.

The case of the patient presented in the current study is similar to previously reported cases with a subacute onset, presence of underlying diabetes and chronic obstructive pulmonary disease. However, the current case differs to previous reports in that the patient mainly had a low-grade fever, with the temperature fluctuating around $37.5^{\circ} \mathrm{C}$, and was in good general condition, radiological examination revealed patchy exudation in the lower lobe, these resulted in a misdiagnosis of pneumonia initially. Therefore, in cases where antibiotic treatment is ineffective and the patient has an underlying disorder, unusual pulmonary infections such as mucormycosis should be considered. In such cases, urgent bronchoscopy should be performed in order to identify any pathogenic organisms and minimize the delay in providing the appropriate treatment. The 
gold standard for the diagnosis of pulmonary mucormycosis is the finding of characteristic hyphae and pathologic changes in a biopsy specimen. The sensitivity of microscopic examination of sputum and sputum culture is low, and the false positive rate is high (17). In the present case, when antibiotic treatment was ineffective, a culture of mucus was obtained by bronchoscopy, which resulted in identification of the pathogenic organisms and thus clarification of the diagnosis.

The majority of published studies state that liposomal amphotericin B is effective against pulmonary mucormycosis $(7,18,19)$. In the present case, plain amphotericin B was used rather than the liposomal form, and the patient did not experience any fever, chills or adverse gastrointestinal reactions. The treatment had a low overall cost and was effective, with a cumulative dosage of $1,355 \mathrm{mg}$ administered over a period of 2 months, resulting in complete recovery and discharge. Furthermore, one of the key aspects for the successful treatment of pulmonary mucormycosis is the management of any risk factors. The current patient had a risk factor of diabetes. During the amphotericin B treatment, subcutaneous injection of insulin was also provided to control and stabilize his blood glucose concentration. In addition, the renal function, serum potassium concentration and temperature of the patient were carefully monitored to maximize the effects of treatment.

\section{References}

1. Liu YN, She DY, Sun TY, Tong ZH, He B, Xiao Y, He LX, Qu JM, Liu XQ, Li ER, et al: A multicenter retrospective study of pulmonary mycosis clinically proven from 1998-2007. Zhonghua Jie He He Hu Xi Za Zhi 34: 86-90, 2011 (In Chinese).

2. Spellberg B, Kontonyiannis DP, Fredricks D, Morris MI, Perfect JR, Chin-Hong PV, Ibrahim AS and Brass EP: Risk factors for mortality in patients with mucormycosis. Med Mycol 50: 611-618, 2012.

3. Yang Y, Fang B, Xu X, Fang F, Pan M, Zhong X and Sun T: Pulmonary mucormycosis: report of 5 cases and review of 46 cases reported in China. Chin J Tuberc Respir Dis 36: 572-576, 2013. (In Chinese)

4. Tedder M, Spratt JA, Anstadt MP, Hegde SS, Tedder SD and Lowe JE: Pulmonary mucormycosis: Results of medical and surgical therapy. Ann Thorac Surg 57: 1044-1050, 1994.

5. Garcia-Covarrubias L, Bartlett R, Barratt DM and Wassermann RJ: Rhino-orbitocerebral mucormycosis attributable to Apophysomyces elegans in an immunocompetent individual: Case report and review of the literature. J Trauma 50 353-357, 2001.
6. Li WF, He C, Liu XF, Wang SY, Qu JL and Lin ZF: A diagnosis neglected for 6 years: Report of a misdiagnosed case of pulmonary mucormycosis and review of the literature. Chin Med J (Engl) 123: 2480-2482, 2010.

7. Wolf O, Gil Z, Leider-Trejo L, Khafif A, Biderman P and Fliss DM: Tracheal mucormycosis presented as an intraluminal soft tissue mass. Head Neck 26: 541-543, 2004.

8. Singh J,Rimek D and Kappe R: In vitro susceptibility of 15 strains of zygomycetes to nine antifungal agents as determined by the NCCLS M38-A microdilution method. Mycoses 48: 246-250, 2005.

9. Sun QN, Fothergill AW, McCarthy DI, Rinaldi MG and Graybill JR: In vitro activities of posaconazole, itraconazole, voriconazole, amphotericin B, and fluconazole against 37 clinical isolates of Zygomycetes. Antimicrob Agents Chemother 46: 1581-1582, 2002.

10. Chen W, Ye Q, Ming Y, Li Z, Xiong L and Ren Z: Diagnosis and treatments of mucor infection after allogenic organ transplantation. Linchuang Waike Zazhi 16: 31-33, 2008 (In Chinese).

11. Chen Lamei and Li Chunyang: Research progress on mucormycosis. Chin J Mycol: 243-246, 2007. (In Chinese)

12. Hamilos G, Samonis G and Kontoyiannis DP: Pulmonary mucormycosis. Semin Respir Crit Care Med 32: 693-702, 2011.

13. Skiada A, Pagano L, Groll A, Zimmerli S, Dupont B, Lagrou K, Lass-Florl C, Bouza E, Klimko N, Gaustad P, et al: Zygomycosis in Europe: Analysis of 230 cases accrued by the registry of the Europe confederation of medical mycology (ECMM) working group on zygomycosis between 2005 and 2007. Clin Microbiol Infect 17: 1859-1867, 2011.

14. Roden MM, Zaoutis TE, Buchanan Wl, Knudsen TA, Sarkisova TA, Schaufele RL, Sein M, Sein T, Chiou CC, Chu JH, et al: Epidemiology and outcome of zygomycosis: A review of 929 reported cases. Clin Infect Dis 41: 634-653, 2005.

15. Liang Z, Jin F, She D and Chen L: Clinical analysis of 5 patients with pathologically diagnosed pulmonary mucormycosis. J Clin Pulm Med: 418-419, 2012. (In Chinese)

16. Yang $\mathrm{C}$ and Xie X: Diabetes and Nose-Cerebral Mucormycosis: A Case Study. Shiyong Yiyuan Linchuan Zachi 12: 183-184, 2015 (In Chinese).

17. The Subspeciahy Group of Infectious Diseases, Society of respiratory, Chinese Medical Association, Editorial board of Chin J Tuberc Respir Dis: Specialist Consensus of Diagnosis and Treatment on Pneumonomycosis. Zhonghua Jihe He Huxi Zachi 30: 821-834, 2007 (In Chinese).

18. Limper AH, Knox KS, Sarosi GA, Ampel NM, Bennett JE, Catanzaro A, Davies SF, Dismukes WE, Hage CA, Marr KA, et al: An official American Thoracic Society statement: Treatment of fungal infections in adult pulmonary and critical care patients. Am J Respir Crit Care Med 183: 96-128, 2011.

19. Spellberg B, Walsh TJ, Kontoyiannis DP, Edwards J Jr and Ibrahim AS: Recent advance in the management of mucormycosis: From bench to beside. Clin Infect Dis 48: 1743-1751, 2009. 